\title{
Premier inventaire faunistique des Chironomidés (Diptera : Chironomidae) des eaux courantes de Tunisie
}

\author{
M. Boumaizal \\ H. Laville2
}

Mots clés : Diptera, Chironomidae, faunistique, biogéographie, Tunisie.

Pour la première fois, une liste faunistique de 81 taxa de Chironomidés - 63 identifiés à l'espèce -récoltés par dérive dans 23 oueds du Nord, 2 oueds et 5 oasis du Sud de la Tunisie est présentée.

La faune est à dominante paléarctique (55 espèces : $87,3 \%$ ). Les $12,7 \%$ restants ( 8 espèces) sont des élérnents de la faune méditerranéenne (3), nord-africaine (2), af rotropicale-ouest paléarctique (2), panpaléotropicalc (1).

De nouvelles récoltes d'imagos et nymphes $\sigma$ matures sont nécessaires pour confirmer l'apparente nouveauté spécifique de 5 types d'exuvies nymphales appartenant aux genres Larsia, Parakiefferiella, Paratanytarsus, Tanytarsus, Virgatanytarsus. 170 especes de Chironomidés sont à présent recensées d'Afrique du Nord.

First faunistic list of chironomids (Diptera : Chironomidae) for running-waters in Tunisia

Kievwords : Diptera, Chironomidae, faunistic, biogeography, Tunisia.

For the first time, a faunal list with 81 taxa of Chironomidae, 63 identified to species, is presented for Tunisia, using tlata from drift samples taken in 23 oueds from the North, 2 oueds and 2 oases from the South.

The faunal list is dominated by palaearctic taxa ( 55 species : 87.3\%). The remaining $12.7 \%$ ( 8 species) are from elements of the Mediterranean fauna (3 species), north Africa (2 species), afrotropical-west palaearctic (2 species), panpalaeotropical (1 species).

New collections of adults and male pupae were necessary to confirm the specific originality of five types of pupal exuviae belonging to the genera Larsia, Parakiefferiella, Paratanytarstu, Tanytarsus, Virgatanytarsus.

170 species of chironomids have been recorded from North Africa up to now.

Dans le cadre de l'étude faunistique des Chirono nidés d'Afrique du Nord, zone de passage entre les régions paléarctique et afrotropicale, un premier inventaire consacré au Maroc a permis de recenser 134 especes (Azzouzi \& Laville 1987). Simultanément, l'étude de récoltes effectuées par l'un de nous (M. Boumaiza) dans 35 stations réparties dans le réseau hydrographique du Nord et du Sud tunisien nous amène à présenter la première liste faunistique

\footnotetext{
1. Faculté des Sciences, Département des Sciences Biologiques, Campus Universitaire, 1060 Tunis, Tunisie.

2. Laboratoire d'Hydrobiologie, UA. 695 C.N.R.S., Université Paul Sabatier, 118 route de Narbonne, 31062 Toulouse Cédex, France.
}

des Chironomidés de Tunisie où, jusqu'ici, une seule espèce halobionte à répartition méditerranéenne, Baeotendipes noclivaga (K.), était signalée.

\section{Méthodes et stations étudiées}

Au cours d'une étude de l'ensemble des peuplements macrobenthiques des eaux courantes - oueds et oasis - de Tunisie dont les Oligochètes (Boumaiza et al 1986) et les Simulidés (Boumaiza \& Clergue 1986) ont fait l'objet de publications récentes, des récoltes de Chironomidés ont été effectuées essentiellement en 1985, puis étudiées au Laboratoire d'Hydrobiologie de Toulouse en 1986-87. 


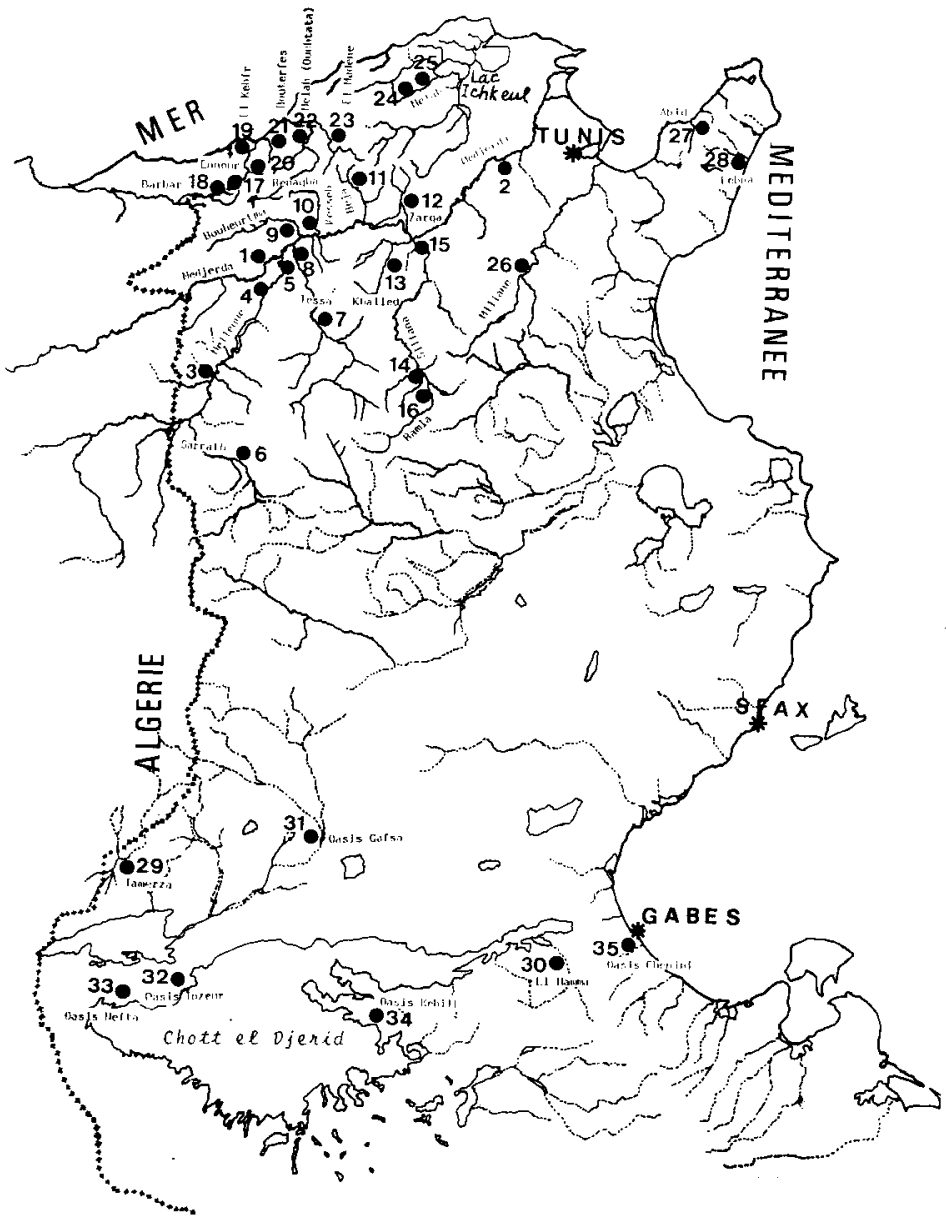

Fig. 1: Localisation des 35 stations d'étude 
Ces récoltes concernent des nymphes, exuvies nymphales, imagos $\sigma$ noyés, recueillis à l'aide de filets à dérive de 200 à $300 \mu \mathrm{m}$ de vide de maille dans 28 stations réparties dans le réseau hydrographique Nord et 7 stations dans les oueds et les oasis du Sud, au niveau d'une ligne Tozeur-Gabés, limite septentrionale du Chott el Djérid (fig. 1).

Chaque station n'a été échantillonnée qu'une seule fois, la majorité (27) en avril 1985.

Les stations prospectées sont numérotées de 1 à 35 sur la carte du réseau hydrographique tunisien (fig. 1): elles se répartissent dans 5 sous-régions géographiques :

- 16 stations dans 11 oueds du Bassin Versant de la Medjerda qui représente le plus important réseau de Tunisie :

-7 stations dans 7 petits oueds du Nord-Ouest (Kroumirie et Mogods) ;

- 2 stations dans le systeme hydrologique du Lac Ichkeul (Nord) :

- 3 stations dans 3 oueds du Nord-Est : Miliane, Abid, Lebna ;

-7 stations ( 2 oueds +5 oasis) dans le Sud.

Les principales caractéristiques physiques de ces stations sont récapitulées sur le tableau I.

Leur situation altitudinale s'échelonne du niveau de la mer (Oued Lebna) a $572 \mathrm{~m}$ (Oued Sarrath, affluent Sud de l'Oued Mellegue).

Dans le Sud, les oasis avec des eaux thermales aux temperatures toujours supérieures à $23^{\circ} \mathrm{C}$ et une alimentation soit par des sources chaudes $(\mathrm{O}$. Nefta, 0 . Tozeur, $\mathrm{O}$. Gafsa) soit par des puits artésiens $(\mathrm{O}$. Kebili) sont des milieux originaux.

Toutes ces stations sont décrites plus en détail dans un précédent travail (Boumaiza 1984) mais avec une numérotation différente récapitulée colonne $\mathrm{N} 2$ du tableau I.

\section{Faunistique et biogéographie}

L'inventaire faunistique présenté est le premier consacré à la Tunisie où une seule espèce Baeoten. dipes noctivaga (K.) était signalée des eaux saumâtres littorales entre Carthage, La Marsa, Korbous, Saint-Germain et Tunis (Wülker 1957a sub Haliella brevimana K., Reiss 1977 sub Haliella noctivaga K. Synonymie du genre Haliella dans Pinder \& Reiss 1986). 81 taxa, tous nouveaux pour la faune tunisienne, ont été recensés dans cette étude : ils se répartissent ainsi dans les différentes sous-familles ou tribus : Tanypodinae $10(12,3 \%)$, Diamesinae 1 $(1,2 \%)$, Prodiamesinae $1(1,2 \%)$, Orthocladiinae 30 (37\%), Chironomini $27(33,3 \%)$. Tanytarsini 12 $(14,8 \%)$.

Le tableau II récapitule leur liste avec leurs différentes stations de récoltes.

Ces récoltes enrichissent de 29 taxa supplémentaires la connaissance de la faune chironomidienne d'Afrique du Nord où 170 espèces sont maintenant recensées : 134 au Maroc, 82 en Tunisie, 20 en Algérie.

- En Algérie, outre les 5 espèces signalées isolément dans la littérature, Paramerina vaillanti Fitt. (Fittkau 1962), Polypedilum aegyptium K., Micropsectra clastrieri (Reiss 1969), Orthocladius (Eudacty. locladius) fuscimanus (K.) (Cranston 1984) et Paratanytarsus mediterranetss Reiss \& Säwedal (Reiss 1985), une liste de 15 espèces a été inventoriée par Reiss (1977) :

Espèces paléarctiques (11)

Orthocladius (Eudact.) fuscimanus (K.)

* Microtendipes britteni Edw. Paracladopelma laminata K. Polypedilum cultellatum $\mathrm{G}$. Stictochironomus pictulus (Mg.) Micropsectra atrofasciata $\mathrm{K}$. Paratanytarsus inopertus (Walk.)

* Paratanytarsus? tenuis (Mg.) Rheotanylarsus muscicola K. Stempellina almi $\mathrm{Br}$. Tanytarsus fimbriatus Reiss \& Fitt.

Espèces méditerranéennes (5)

Paramerina vaillanit Fitt.

* Halocladius stagnorum (G.) Virgatanyuarsus maroccanus Kugl. \& Reiss

* Micropsectra clastrieri Reiss Paratanytarsts mediterraneus Reiss \& Säwedal

Espèces afrotropicales (3) Chironomus calipterus $\mathbf{K}$.

* Dicrotendipes peringueyanus $\mathrm{K}$. Tanytarsus horni $\mathrm{G}$.

Espèce afrotropicale - ouest paléarctique (1) Polypedilum aegyptium $\mathrm{K}$.

Les 5 espèces précédées d'un astérisque sont seulement connues d'Algérie. 
Tableau I. Caractéristiques physiques des stations d'étude.

$\mathbf{T}^{\circ} \max$. (Juin-Juillet); $\mathbf{T}^{\circ}$ min. (Novembre-Janvier).

Nature du courant : $m$; modéré; $r$ : rapide; 1 : lent.

Substratum : $\mathbf{P}$ : pierres ou galets; $\mathbf{S}$ : sable ; $\mathbf{B}$ : blocs ; $\mathrm{G}$ : graviers.

N2 : numérotation des stations d'aprés Boumaiza (1984).

\begin{tabular}{|c|c|c|c|c|c|c|c|c|c|}
\hline $\mathrm{N}_{1}$ & $\mathrm{~N}_{2}$ & & 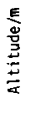 & $\begin{array}{l}\dot{E} \\
\stackrel{E}{U} \\
\dot{D}\end{array}$ & \begin{tabular}{l}
$\dot{x}$ \\
0 \\
U \\
0 \\
0 \\
\hdashline
\end{tabular} & 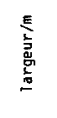 & 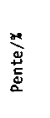 & 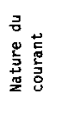 & 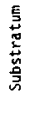 \\
\hline 1 & (1) & Medjerda I (Jendouba) & 146 & & $24^{\circ}$ & $>10$ & 0,2 & m & s \\
\hline 2 & (2) & Medjerda I: (Bathan) & 28 & $6^{\circ}$ & $24^{\circ}$ & 10 & 0,2 & $r$ & P \\
\hline 3 & (14) & 0. Mellegue I & 329 & $13^{\circ}$ & $26^{\circ}$ & $>10$ & 0,3 & $m$ & $s$ \\
\hline 4 & $(15)$ & 0. Meliegue II & 169 & & $26^{\circ}$ & $>10$ & 0,1 & m & $\mathbf{p}$ \\
\hline 5 & $(16)$ & 0. Mellegue III & 138 & & $20^{\circ}$ & $>10$ & 0,1 & $r$ & $s$ \\
\hline 6 & & 0. Sarrath & 572 & & & $<4$ & 0,3 & $r$ & $\mathbf{P}$ \\
\hline 7 & $(12)$ & 0. Tessa I cours > & 295 & & $23^{\circ}$ & $>6$ & 0,3 & $m$ & s \\
\hline 8 & $(13)$ & 0 . Tessa II cours < & 130 & & & $>10$ & 0,2 & $r$ & s \\
\hline 9 & (7) & 0. Bouheurtma & 130 & & $23^{\circ}$ & $>6$ & 0,1 & $m$ & $P$ \\
\hline 10 & (6) & 0. Kesseb & 134 & $9^{\circ}$ & $22^{\circ}$ & $>6$ & 0,1 & r & $P$ \\
\hline 11 & (4) & 0. Beja 1 & 155 & & $23^{\circ}$ & $4-6$ & 0,5 & $r$ & B \\
\hline 12 & (3) & 0. Zarga & 95 & $8^{\circ}$ & $23^{\circ}$ & $<5$ & 0,2 & $\mathbf{r}$ & $P$ \\
\hline 13 & $(11)$ & 0. Khalled & 230 & $11^{\circ}$ & $19^{\circ}$ & $2-3$ & 1,3 & $\mathbf{r}$ & $\mathrm{P}$ \\
\hline 14 & (9) & 0. Siliana I (Siliana) & 420 & $16^{\circ}$ & $26^{\circ}$ & $<4$ & 1,5 & $\mathbf{r}$ & $P$ \\
\hline 15 & $(10)$ & 0. Siliana $\vee$ (Testour) & 78 & $14^{\circ}$ & $21^{\circ}$ & $<4$ & 0,3 & $r$ & $P$ \\
\hline 16 & & 0. Ramla & 470 & & & $<4$ & 0,7 & $m$ & P \\
\hline 17 & $(18)$ & o. Ennour & 400 & $11^{\circ}$ & $27^{\circ}$ & $<4$ & 4,5 & m & B \\
\hline 18 & & 0. Barbar & 128 & & & 4 & 0,4 & $\mathbf{r}$ & B \\
\hline 19 & $(19)$ & 0. El Kebir (Tabarka) & 8 & & $30^{\circ}$ & $>4$ & 0,1 & $r$ & s \\
\hline 20 & $(20)$ & 0. Renagha & 65 & & $26^{\circ}$ & $<4$ & 1,0 & $m$ & p \\
\hline 21 & $(21)$ & 0. Bouterfes & 23 & & $25^{\circ}$ & $>4$ & 0,7 & $r$ & P \\
\hline 22 & (22) & 0. Melah (Ouchtata) & 29 & & & $>10$ & 0,2 & $m$ & s \\
\hline 23 & $(23)$ & 0. El Madène & 30 & & & $>6$ & 0,4 & $r$ & $\mathbf{p}$ \\
\hline 24 & $(27)$ & 0. Melah I (Djalta) & 15 & $11^{\circ}$ & $26^{\circ}$ & $<4$ & 0,2 & $r$ & B \\
\hline 25 & & 0. Melah II (Teskraia) & 10 & & & $>10$ & 0,1 & $\mathbf{r}$ & $P$ \\
\hline 26 & $(30)$ & 0. Miliane I (El Fahs) & 168 & & $26^{\circ}$ & $>4$ & 0,2 & m & $\mathbf{p}$ \\
\hline 27 & (33) & 0. Abid II & 12 & $10^{\circ}$ & $30^{\circ}$ & $4-6$ & 0,2 & 1 & $s$ \\
\hline 28 & (34) & 0 . Lebna (Lebna) & 7 & & $27^{\circ}$ & 6 & 0.2 & 1 & $s$ \\
\hline 29 & & 0. Tamerza & 300 & & $30^{\circ}$ & $<3$ & 1,5 & $m$ & $s$ \\
\hline 30 & $(41)$ & 0. El Harma & 52 & $12^{\circ}$ & $20^{\circ}$ & $<3$ & 0.2 & 1 & s \\
\hline 31 & $(42)$ & Oasis Gafsa & 290 & $23^{\circ}$ & $30^{\circ}$ & $<2$ & 1,0 & $r$ & s \\
\hline 32 & (44) & Oasis Jozeur & 50 & $23^{\circ}$ & $30^{\circ}$ & $1-3$ & 0.6 & $m$ & $\mathrm{~s}$ \\
\hline 33 & $(45)$ & Oasis Nefta & 75 & $23^{\circ}$ & $30^{\circ}$ & $<3$ & 3,7 & $m$ & $s$ \\
\hline 34 & (46) & Oasis Kebili & 41 & $20^{\circ}$ & $24^{\circ}$ & $<3$ & 3,3 & $m$ & s \\
\hline 35 & (47) & Oasis Chenini & 25 & $13^{\circ}$ & $25^{\circ}$ & $1-2$ & 0.2 & $m$ & $\mathrm{~s}$ \\
\hline
\end{tabular}


- En Tunisie, sur les 81 taxa dénombrés, 63 ont pu être identifiés à l'espèce : $55(87,3 \%)$ présentent une répartition paléarctique, les autres ont une répartition méditerranéenne (3), nord-af ricaine (2), afrotropicale-ouestpaléarctique (2), panpaléotropicale (1):

- Espèces méditerranéennes

"Krenosmittia " hispanica

Parakiefferiella sp d Wülker 1957b

Virgatanyarsus maroccanus

- Espèces nord-africaines

Paramerina vaillanti

Rheomus yahiae

Espèces afrotropicale-ouestpaléarctiques

Polypedilum aegyptium

Kloosia pusilla

- Espèce panpaléotropicale

Chironomus calipterus

L'originalité apparente de 5 types d'exuvies ne pourra être confirmée qu'à partir de nouvelles récoltes d'imagos or et de nymphes or matures dans les stations concernées :

- Larsia sp 1 (Tamerza)

- Parakiefferiella sp d Wülker 1957 b (Medjerda)

- Tanytarsus sp 1 (Chenini)

- Paratanytarsus sp 1 (Tunisie)

- Virgatanytarsus sp 1 (Medjerda)

Le tableau ci-dessous récapitule les nombres d'espèces de chaque sous-familles et tribus récoltés dans les 3 principales sous-régions géographiques visi. tées : Bassin Versant de la Medjerda (50 especes), Oueds cótiers du Nord-Ouest et du Nord-Est (39 espèces), Oueds ct Oasis du Sud (23 espèces).

\section{Espèces intéressantes}

Paramerina vaillanti Fitt.

Récoltée pour la première fois dans 2 Oasis algériennes l'O. Beni Abbes (Nord-Sahara) et l'O. Ham. mada (Hoggar) dont la température atteignait $23^{\circ} \mathrm{C}$ le 3 avril 1955 (Fittkau 1962).
Sa récolte dans le Sud tunisien, dans l'Oasis Chenini près de Gabès $\left(T^{\circ} 25^{\circ} \mathrm{C}\right.$ le 17.04.83), confirme l'écologie de cet te espèce à la fois limnokrène et thermophile. Il s'agit d'une espèce à répartition nordafricaine.

" Krenosmittia " hispanica Wülker 1957 b.

Wülker (1957 b) décrit et signale cette espèce de 2 rivières d'Espagne, l'une du Sud-Ouest (Rio Manol) et l'autre du Sud, à l'Ouest de Pizarra (Sierra Prieta, Malaga). Les 2 stations tunisiennes concernent 2 rivières côtières du Nord : l'Oued Bouterfes $(25 \mathrm{~m})$ et l'Oued El Madène $(30 \mathrm{~m})$.

Sa présence en Espagne et aussi en Italie? (Rossaro 1982) suggère une répartition méditerranéenne.

La nymphe présente une corne thoracique et un segment anal différents de ceux des autres représentants du genre Krenosmittia aussi, sa position générique doit-elle être révisée.

Parakiefferiella sp d Wülker 1957 b

Wülker (1957 b) décrit cette exuvie originale d'après des récoltes dans deux rios d'Espagne: le Rio Guadarrama au Nord-Ouest de Madrid et un rio de la Sierra Prieta à l'Ouest de Malaga.

En Tunisie, les 10 stations connues de cette espèce se répartissent dans le Bassin Versant de la Medjerda (3), dans les oueds du Nord-Ouest (6) et dans l'Oued Tamerza au Sud (1).

\section{Rheosmittia spinicornis ( $\mathrm{Br}$.)}

Répartition : Suède, Danemark (Cranston \& Saether 1986), Highlands d'Ecosse (Langton 1984), Cours alpin du Rhin (Wilson \& Wilson 1985), Alpes de Haute Bavière $(620 \mathrm{~m})$ (Reiss 1982), Carpathes roumaines : Vallée d'Olt et Monts Lotru (Cure 1985), Pyrénées : Gave de Bious (1600 m) (Laville \& Vinçon 1986).

Cette espèce à répartition ouest-paléarctique est citée pour la première fois d'Afrique du Nord dans deux affluents S.O. de la Medjerda: I'O. Mellegue $(140 \mathrm{~m})$ et l'O. Tessa II $(130 \mathrm{~m})$. Elle parait coloniser aussi bien le potamal des grandes rivieres que le

\begin{tabular}{lccccccc}
\hline & Tanyp. & Diam. & Prod. & Orthoc. & Chiro. & Tanyt. & Total \\
\hline B.V. Medjerda & 7 & 1 & - & 18 & 17 & 7 & 50 \\
Oueds N.O. \& N.E. & 4 & - & 1 & 21 & 7 & 6 & 39 \\
Oueds + Oasis S & 3 & - & - & 10 & 7 & 3 & 23 \\
\hline
\end{tabular}




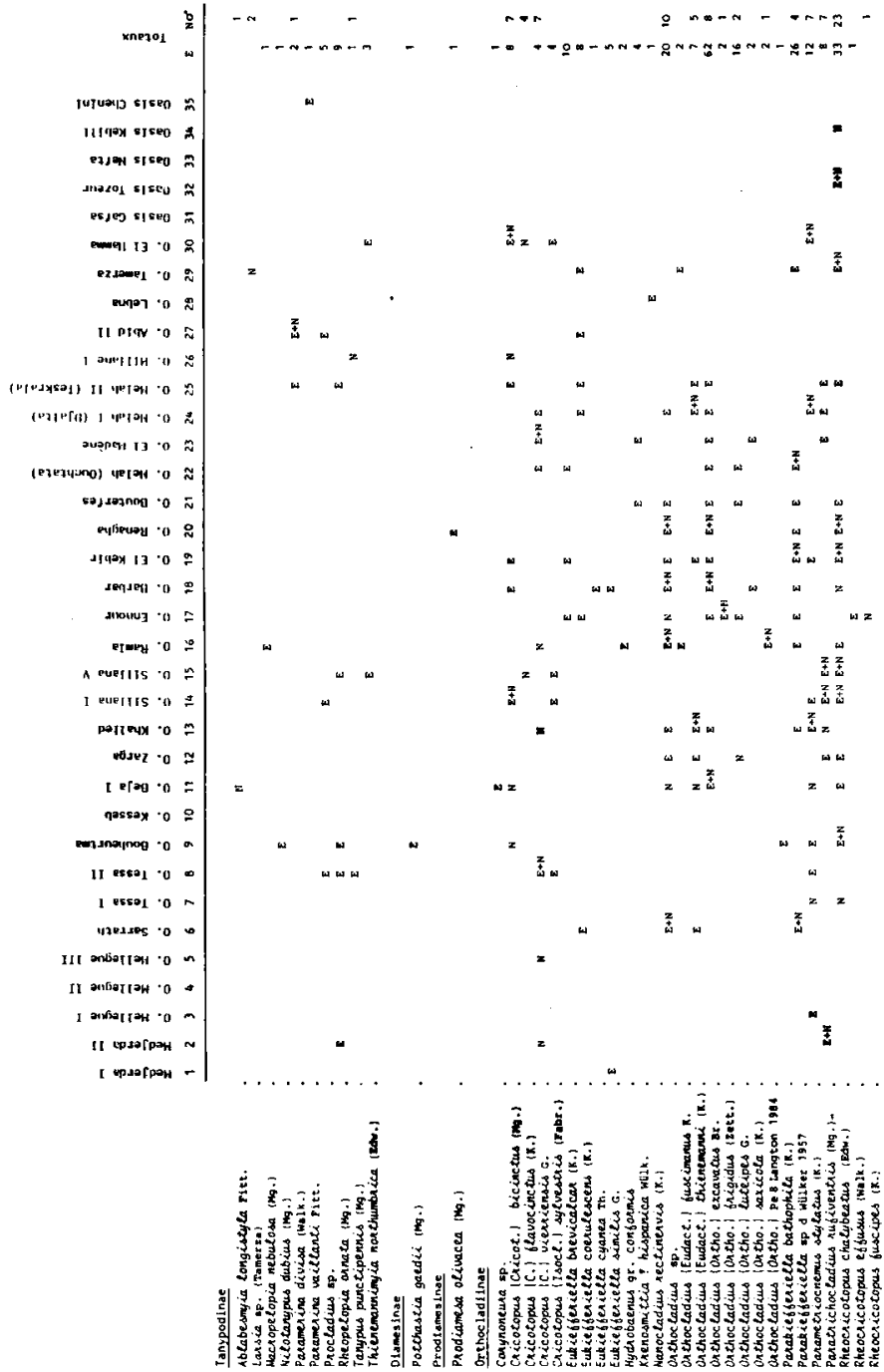




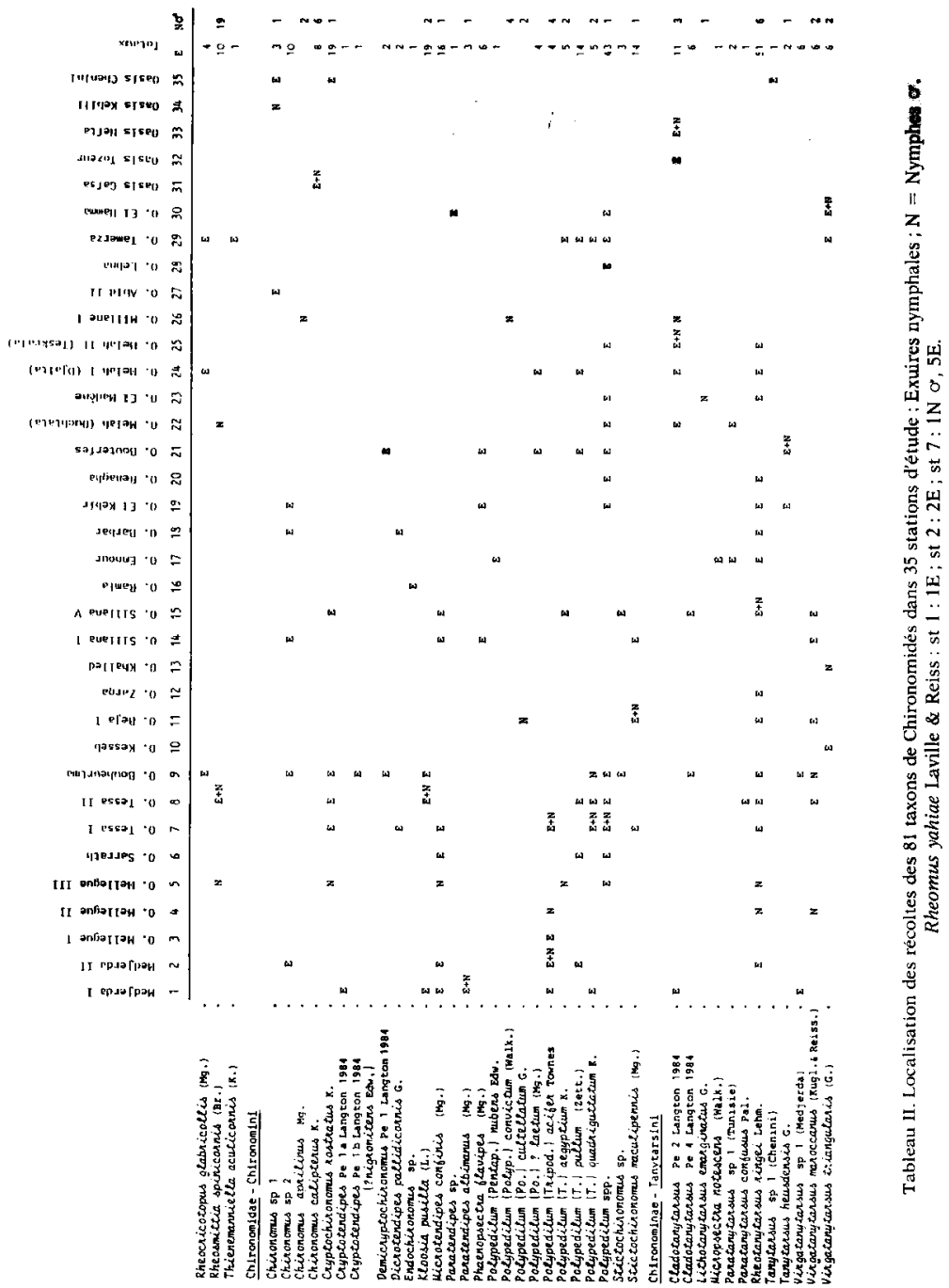


rhithral des rivières de montagne comme le Gave de Bious $(1600 \mathrm{~m})$ dans les Pyrénées atlantiques.

\section{Chironomus calipterus Freem.}

Cet te espèce à vası répartition panpaléotropicale est signalee jusqu'au Pakistan et au N.O. de la Thailande (Reiss 1977), dans toute la région circumméditerranéenne - Grèce, Algérie, Maroc, SudEspagne, Sud-France, Egypte, Israël, Turquie (Reiss 1977), Syrie (Reiss 1986) -, dans toute l'Afrique et Madagascar (Freeman 1957).

\section{Kloosia pusilla (L.)}

Répartition ouest-paléarctique : ? Norvège, Hollande, Roumanie, Pologne, URSS et région méditerranéenne - Italie (moyen Pô), Grèce, Turquie, Tunisie - (Reiss 1988).

Il s'agit d'un élément faunistique du potamal dont les larves vivent dans les sédiments sableux des plus grands fleuves à faible courant et pauvres en matière organique.

En Tunisie, elle a été récoltée dans la Medjerda et dans l'un de ses affluents N-O, l'Oued Bouheurtema, ainsi que dans deux Oasis méridionales, Tozeur et Nefta.

\section{Polypedilum acifer Townes}

Signalée du Nord des U.S.A., dans le Michigan (Townes 1945), elle est également connue de la région sud-paléarctique : Sud-Yougoslavie, Grèce, Maroc, Roumanie (Reiss 1977).

Récentes récoltes dans 3 oueds marocains du Nord (O. Fès, O. Boufekrane, O. Sebou) (Azzouzi \& Laville 1987) et dans 3 oueds tunisiens du Nord (O. Medjerda, O. Mellegue, O. Tessa).

\section{Polypedilum aegyptium K.}

Syn. pruina Freem., vetterense Br. par Albu (1980) Syn, airense Freem.

Connue des régions ouest-paléarctique et afrotropicale : largement répandue dans presque toute l'Europe, l'Asie mineure, le Sud de l'U.R.S.S.

Pourtour méditerranéen : Espagne, Pyrénées $\alpha$ cidentales, Grèce, Turquie, Egypte, Algérie, Maroc (Reiss 1985). Semble présenter la même aire de distribution que Microchironomus deribae (Freem.).

En Tunisie du Nord, elle a été récoltée dans 2 oueds - Mellegue et Siliana - affluents rive droite de la Medjerda et dans l'Oued Tamerza au Sud.

\section{Rheomus yahiae Laville \& Reiss 1988}

Espèce nord-africaine connue du Maroc dans l'Oued Fès (Az\%ouzi \& Laville 1987) et de Tunisie dans l'O. Medjerda et dans son affluent l'O. Tessa, $2 \mathrm{~km}$ en amont de leur confluence.

Elément faunistique du potamal rencontré en compagnie de Kloosia pusilla dans l'Oued Medjerda.

Virgatanytarsus maroccanus Kugl. \& Reiss

Répartition méditerranéenne: Maroc, Algérie (Kügler \& Reiss 1973, fig. 16), Crète (Reiss 1977).

Stations d'Afrique du Nord:

- Algérie : $\mathbf{4}$ stations : Cascade de Bérard (Locus typicus), Biskra, Blida, Oued Béchar ;

- Maroc : 2 stations : dans le Moyen Atlas - Oued Oum-er-Rbia amont - et dans le Haut Atlas M'Hamid Dra-Tal - (Azzouzi \& Laville 1987);

- Tunisie : Réseau Medjerda : 3 affluents RD - 0 . Mellegue, Tessa, Siliana - et 2 affluents RG - O. Bouheurtema, Beja -

\section{Virgat anytarsus triangularis (G.)}

Signalée du Nord de l'Europe - de l'Irlande à la Roumanie - (Kügler \& Reiss 1973, fig. 16) et plus récemment dans le Sud - Massif Central Français (Laville \& Serra Tosio 1987), Espagne (Rieradevall 1985), et en Asie Mineure - Turquie (Reiss 1985) -

Premiere citation sur le continent africain.

Stations tunisiennes: Dans le Nord, 2 affluents de l'O. Medjerda, l'O. Kesseb (RD) et l'O. Khalled (RG), et dans le Sud, l'O. Tamerza et l'O. el Hamma.

\section{Conclusion}

Réalisé dans le cadre d'une étude faunistique de l'ensemble des macroinvertébrés des eaux courantes, essentiellement de plaine, de Tunisie, ce premier inventaire Chironomidien a permis d'identifier une faune de 63 espèces à dominante paléarctique avec quelques éléments à répartition méditerranéenne (3) ou nord-africaine (2).

Si le matériel récol té par dérive permet l'identification rapide d'un grand nombre d'especes dont le stade nymphal est connu, il s'avère insuffisant, notamment pour 5 types d'exuvies non identifiées ; seules de nouvelles captures d'imagos $\sigma$ et de nymphes $\sigma$ matures permett ront d'en préciser l'apparente nouveauté. 
Il est apparu au cours de cette étude que les grandes rivières de plaine du type Medjerda et ses affluents ainsi que les Oasis du Sud à la limite du Sahara abritent une faune psammophile voire thermophile des plus originale.

C'est vers ces types de milieux qu'il nous parait souhaitable d'orienter les futures recherches faunistiques et écologiques des Chironomidés de Tunisie.

\section{Travaux citès}

Albu (P.). 1980. - Indexul sistematic al speculor din Romania. Ordinul Diptera familia Chironomidae Meigen sub familia Chironominae. In Fauna Republici socialiste Romania., vol. XI., fasc. $13: 82-307$.

Artouzi (A.) \& Laville (H.). 1987. - Premier inventaire Eaunistique des Chironomidés (Diptera, Chirononidae) du Maroc. Annls Limnol., 23 (3) : 217.224.

Boumaiza (M.). 1984. - Contribution à la limnologie de la Tunisie. Etude physico-chimique. Archs Inst. Pasteur, Ttmis, 61 (2-3) : 205-246.

Boumaiza (M.) \& Clergue-Gazeau (M.). 1986. - Le peuplement simulidien de la Tunisie: I. Inventaire faunistique et biogéographie (Diptera, Nematocera). Annls Limmol, 22 (1): 31.39.

Boumaiza (M.), Martinez-Ansemil (E.) \& Giani (N.). 1986. - Les Oligochètes et Aphanoneura des eaux courantes de Tunisie. I. Données faunistiques. Annls Limnol. 22 (3) : 231-237.

Cranston (P.S.). 1984. - The taxonomy and ecology of Orhocladius (Eudact ylacladius) ftuscimanus (Kieffer), a hygropetric chironomid (Diptera). J. Nat. Hist. $18: 873-895$.

Cranston (P.S.) \& Saether (O.A.). 1986. - Rheosminia (Diptera : Chironomidae) : a generic validation and revision of the western Palearctic species. J. Nat. Hist., 20:3!-51

Cure (V.). 1985. - Chironomidae (Diptera-Nematocera) aus Rumänien unter besonderer Berücksichtigung jener aus dem hydrographischen Einzugsgebiet der Donau. Arch. Hydrobiol. Sitppl., 68: 163-217.

Fittkau (E.J.). 1962. - Die Tanypodinae (Diptera, Chironomidae). Abh. Larvalsyst. Insekten, $6: 1-453$.

Freeman (P.). 1957. - A study of the Chironomidae (Diptera) of Africa south the Sahara. Ill. Bull. Br. Mis. nat. Hist., Ent., 5 : 323-426.

Kügler (J.) \& Reiss (F.) 1973. - Die triangularis - Gruppe der Gat. tung Tanytarsts v.d.W. (Chironomidae, Diptera). Ent. Tidskr., $94: 59-82$.

Langton (P.H.). 1984. - A key to pupal exuviae of British Chironomidae. Privately printed : $324 \mathrm{pp}$.
Laville (H.) \& Reiss (F.). 1988. - Rheomts, un nouveau genre du complexe Harrischio avec deux nouvelles especes d'Af rique du Nord (Diplera, Chironomidae). Spixiana, Suppl.

Laville (H.) \& Serra-Tosio (B.). 1987. - Chironomides (Diptera) du Massif Central el des basses régions avoisinantes. Amnls Limnol., 23 (2): 135-145.

Laville (H.) \& Vinçon (G.). 1986. - Inventai re 1986 des Chironomidés (Diptera) connus des Pyrénées. Annls Limnol., 22 (3) : 239-251.

Pinder (L.C.V.) \& Reiss (F.). 1986. - The pupae of Chironominae (Diptera : Chironomidae) of the Holarctic region - Keys and diagnoses. Ent. scand. Suppt., $28: 299-47 !$.

Reiss (F.). 1969. - Revision der Gattung Micropsectra Kieff., 1909 (Diptera, Chironomidae) 1. Die al tenuata - Gruppe der Gat tung Micropsectra. Beschreibung 5 neuer Arten aus Mitteleuropa und Nordafrica. Disch. Ent. Z. 16 (IV-V) : 431-449.

Reiss (F.). 1977. - Verbreitungsmuster bei paläarktischen Chironomidenarten (Diptera, Chironomidae). Spixiand, 1: 85-97.

Reiss (F.). 1982. - Die Chironomidenfauna der Murnauer Mooses in Oberbayern (Insecta, Diptera). Entomofama, Zeitschrift fïr Entomologie, Stuppl. 1:263-288.

Reiss (F.). 1985. - Contribution to the zoogeography of the turkisch Chironomidae (Diptera). Isr. J. Entomol., 19: 161-170.

Reiss (F.). 1986. - Ein Beitrag zur Chironomidenfauna Syriens (Diptera, Chironomidae). Entomofauna, Zeirschrift fïr Entomologie, 7 (11): 153-168.

Reiss (F.). 1988. - Die Gattung Kloosia Kruseman, 1933 mit der Neubeschreibung zweier Arten (Chironomidae, Diptera). Spixiana, Suppl.

Rieradevall (M.). 1985. - Ritme diari de la deriva en una estacio del riu Llobragat, amb especial atencio a les exuvies pupals dels Chironomidae (Dipt.). Tesi de Llicencialura, Barcelona : $175 \mathrm{pp}$.

Rossaro(B.). 1982. - Guide per il riconoscimento delle specie antimali delle acque inlerne italiane. 16. Chironomini, 2 (Diptera, Chinonomidac: Orthocladiinat). Collana del progetto finalizzato * Promozione della qualita dell'-ambiente *. $\mathrm{AQ} / 1 / 171$. Coordinatore: S. Ruffo: $80 \mathrm{pp}$.

Townes, Jr. (H.K.). 1945. - The nearctic species of Tendipedini (Diptera, Tendipedidae) (= Chironomidae). Am. Midl. Nat., 34 : $1-206$.

Wilson (R.S.) \& Wilson (S.E.). 1985 . - A survey of the distribution of Chironomidae (Diptera, Insecta) of the river Rhine by sampling pupal exuviae. Hydrobiol. Bull. $18(2)$ : 119-132.

Wülker (W.). 1957a. - Eine spanische Haliella (Dipt, Chironomidae) Arch. Hudrobiol, Suppl., 24 : 281.296.

Wüker (W.). 1957b. - Cber die Chironomiden der Parakiefferiella Gruppe (Diptera : Tendipedidae: Orthocladinac). Beilr. Ent. $7: 411-429$. 